\title{
On Generating Random Network Structures: Trees $^{\star}$
}

\author{
Alexey S. Rodionov ${ }^{1}$ and Hyunseung $\mathrm{Choo}^{2}$ \\ 1 Institute of Computational Mathematics and Mathematical Geophysics \\ Siberian Division of the Russian Academy of Science \\ Novosibirsk, RUSSIA +383-2-396211 \\ alrod@rav.sscc.ru \\ 2 School of Information and Communication Engineering \\ Sungkyunkwan University \\ 440-746, Suwon, KOREA +82-31-290-7145 \\ choo@ece.skku.ac.kr
}

\begin{abstract}
Random trees (RTs) are widely used for testing various algorithms on tree-type networks and also for generating connected graphs similar to real nets. While random topologies based on RTs are generally accepted as network models, the task of their generation is almost unexplored. In this paper we discuss the set of basic algorithms for generating random trees. The fast algorithms with proven properties are presented for generating random trees under conditions for given restrictions, such as a limited node degree, fixed node degrees, and different probabilities of edge existence. Generating random graphs similar to physical networks are underway.
\end{abstract}

\section{Introduction}

In this paper we present the set of basic algorithms for generating random trees (RTs). RTs are used for testing various algorithms on tree-structural networks and as a base for further algorithms for generating connected graphs. Random graphs are widely used as a model of communication networks, usually for the testing of control algorithms 1,2,3. Using real network structures for an algorithm testing is impossible with a simple reason: to achieve reliable results the large number of different structures with given properties is necessary while actual data for real network structures are usually inaccessible and the number of such real structures is small. While random trees and graphs are widely used as a network model, the task of their generation is almost unexplored. It seems that most authors who use random trees and graphs as a model consider the task as an obvious one. Still there are a lot of problems in the task of random graphs generation, especially when complex limitations are loaded on the graph structure. The usual requirements are to guarantee the uniformity on the given

\footnotetext{
* This paper was partially supported by BK21 program. Dr. H.Choo is the correspondent author
} 
space of graphs (to the enumeration) and attainability of all graphs from the given space. Hereafter we discuss some base approaches for the generation of RTs and present some effective algorithms. The rest of the paper is organized as follows. Section 2 is devoted to the base approaches, notations and concepts. In Section 3 we discuss about the generation algorithms of RTs for equal probabilities of edge existence. Section 4 presents the algorithm for generating RTs with different probabilities of edges existence. Section 5 is a brief conclusion.

\section{Preliminaries}

The variable types are clear from the context usually, or are stated in comments. The following procedures are used in the algorithms descriptions:

- $\operatorname{INC}(\mathrm{n}), \operatorname{DEC}(\mathrm{n})$ - increment and decrement operators;

- FindPlace $(x, V, O K)$ - integer function, returns the index of the first element in vector $\mathrm{V}$ equal to $\mathrm{x}$. OK is TRUE if such element is found and FALSE in the opposite case;

- $\operatorname{remove}(\mathrm{x}, \mathrm{V})$ - removes element $\mathrm{x}$ from vector $\mathrm{V}$ (the last element replace $\mathrm{x})$. If $\mathrm{x}$ is not belongs to $\mathrm{V}$ then no action is done;

- $\operatorname{rand}()$ - random number uniformly distributed on $(0,1)$;

- $\operatorname{random}(\mathrm{n}, \mathrm{m})$ - integer random number uniformly distributed on $[n, n+$ $1, \ldots, m], n \leq m$;

- $\operatorname{random}(\mathrm{P}, \mathrm{n}, \mathrm{m})$ - integer random number distributed on $[n, n+1, \ldots, m]$, $n \leq m$ with probabilities $p_{i}=P\left(x_{i}=n+i-1\right), i=1,2, \ldots, m-n+1$;

- Call Alg (Input,Output) - Call the algorithm or procedure Alg with input Input and output Output.

Let us denote arbitrary non-oriented graph with $N$ nodes and $M$ edges and without multiple edges as $G(N, M)$. Several approaches can be used for the random graphs generation. First and most inefficient approach (but still widely used) is the trial method. A random graph with given number of nodes (and possibly edges) is generated and then its properties are checked to answer the given limitations. As the number of possible $G(N, N-1)$ graphs (with all possible enumerations of nodes) is

$$
S=\left(\frac{N(N-1)}{N-1}\right) \frac{N(N-1)}{2} !=\frac{\left[\frac{N(N-1)}{2} !\right]^{2}}{(N-1) !\left[\frac{N(N-1)}{2}-N+1\right] !},
$$

while the total number of all $N$-node trees (with all possible enumerations of nodes also) is only $N^{N-1}$, the trial method is obviously ineffective for RT generation.

Next basic approach is the "sequential growth": each new element is added to the generated graph without violating the limitation and with guaranteeing its properties. Once more there are two possibilities: either to use trial or "smart" method on each step. We use the sampling without repetition for trial method. 
As a "smart" method we use the method of admissible choice (MAC). This is the method in which on each step the choice is done only from the set of elements that 1) keeps the graph in a given class and 2) does not allow to break any limitation. Let us denote:

- $X_{i}$ - set of nodes in the generated graph on the $i$-th step;

- $E_{i}$ - set of edges in the generated graph on the $i$-th step;

- $A_{i}$ - set of edges that are allowed to be added to the generated graph on the $i$-th step;

- $I n_{i}-$ set of edges that are to be added to $A_{i}$ before the $(i+1)$-st step;

- $E x_{i}$ - set of edges that are to be excluded from $A_{i}$ before the $(i+1)$-st step;

- $v_{i}-i$-th node (vertex) of a graph;

- $e_{i j}$ - edge that connects $v_{i}$ and $v_{j}$;

- $X(G), E(G)$ - sets of nodes and edges of the graph $G$;

- $C(N)$ - the complete graph with $N$ nodes.

Thus, $A_{i+1}=A_{i} \backslash E x_{i} \cup I n_{i}$. Some limitations can lead to the empty $A_{i+1}$. In this case the rollback is needed by one step. If $e_{s t}$ is the last edge chosen before the deadlock then it transfers from $A_{i}$ to $E x_{i}$.

The simple example is to generate random trees in the case of equal probability of an edge existence. If the trial method is used, then after each new edge selection the test is done if addition of this edge makes a cycle or multi-edge in a tree. If yes then the edge is rejected and choice repeats. If MAC is used then first we choose uniformly any node as a root, let it be $v_{f}$. Thus

$$
A_{1}=\left\{e_{f 1}, e_{f 2}, \ldots, e_{f f-1}, e_{f f+1}, \ldots, e_{f N}\right\} .
$$

If on the $i$-th step the edge $e_{s t}$ is selected ( $t$-th node is new) then

$$
E x_{i}=\left\{e_{c t} \mid v_{c} \in X_{i}\right\}, \quad I n_{i}=\left\{e_{t d} \mid v_{d} \in \bar{X}_{i}\right\} .
$$

\section{Algorithms}

First we formally describe the algorithm for generating random trees sketched above and then discuss about more complex cases of random trees with limitations.

\subsection{The Algorithm for Generating Random N-Vertex Trees}

For generating random $N$-vertex trees (the isomorphism is possible) the following fast algorithm is offered:

\section{Fast Random Tree Generating Algorithm.1}

Input: $N$ is the number of nodes.

Output: $O U[1 . . N-1]$ and $I O[1 . . N-1]$ are the vectors of numbers starting and ending nodes for the resulting tree edges ( $i$-th edge connects node $O U_{i}$ with $\left.I O_{i}\right)$.

${ }^{1}$ Some algorithms discussed in this paper including this one were first programmed for the application package Graph-ES, see 4 5.6. 
1. for $i:=1$ to $N$ do

2. $I[i]:=i$;

\Initial vector of node numbers

3. endfor;

4. $1:=\operatorname{random}(1, N)$;

II Choose the root

5. K:=I[N]; I[N]:=I[I]; I[l]:=K; \\Replacement with the last node

6. for $m:=1$ to $N-1$ do

7. $t:=\operatorname{random}(1, \mathrm{~m})$;

\\ New node

8. $\mathrm{s}:=\operatorname{random}(\mathrm{m}+1, \mathrm{~N})$;

\\ Node of "growth"

9. $\mathrm{OU}[\mathrm{m}]:=\mathrm{I}[\mathrm{t}] ; \mathrm{IO}[\mathrm{m}]:=\mathrm{I}[\mathrm{s}] ; \backslash \backslash$ Edge registration

10. $\mathrm{K}:=\mathrm{I}[\mathrm{t}] ; \mathrm{I}[\mathrm{t}]:=\mathrm{I}[\mathrm{N}-\mathrm{m}] ; \backslash \backslash$ Replacement: from $\mathrm{N}-\mathrm{m}+1$ to $\mathrm{N}-$ $\mathrm{I}[\mathrm{N}-\mathrm{m}]:=\mathrm{K}$;

$\backslash$ nodes that are in a tree already

11. endfor.

Remark: It is clear that this algorithm needs only $2 N-1$ calls to the random number generator and uses the minimum memory $(N+4$ integers for the case of equal probabilities and $N+1$ reals additionally in the case of different probabilities of edge existence).

The following theorem is proved for this algorithm.

Theorem 1. Fast Random Tree Generating Algorithm creates any of $N$-vertex tree with equal probability $1 / N^{N-1}$.

Proof is rather obvious but tiresome. At first the equal probability of entire results is proved and then it is proved that the number of different results is equal to $N^{N-1}$. As it is the well-known number for different $N$-node trees (all renumbering included), the attainability is proved also. For the complete proof, refer to $[7$.

\subsection{Generating Trees with the Restriction on a Maximum Node Degree}

Let us have the limitation on the maximum degree of nodes in a tree: $\operatorname{deg}\left(v_{i}\right) \leq$ $D e g$. This leads to the new definition of $E x_{i}$ in comparison with the previous case:

$$
E x_{i}= \begin{cases}\left\{e_{c t} \mid v_{c} \in X_{i}\right\} & \text { if } \operatorname{deg}\left(v_{s}\right) \leq \operatorname{Deg} \\ \left\{e_{c t} \mid v_{c} \in X_{i}\right\} \bigcup\left\{e_{s d} \mid v_{d} \in \bar{X}_{i}\right\} & \text { otherwise. }\end{cases}
$$

The addition of new edges is similar to the case of the unconditional random tree generation, but the set of nodes that are in the tree already is divided into two parts: those with maximum degree $\left(N_{m}\right)$ and those with degrees under it $\left(N_{<}\right)$. New edge must be chosen to connect some free node with the one in $N_{<}$. The algorithm looks as follows:

\section{Algorithm for Generating Trees with Limited Node Degrees (GTD).}

Input: $N$ is the number of nodes; Deg is the possible maximum degree of nodes. Output: $O U[1 . . N-1]$ and $I O[1 . . N-1]$ are the vectors of starting and ending nodes for the resulting tree edges, respectively ( $i$-th edge connect node $O U_{i}$ with $\left.I O_{i}\right)$, Degrees $(1 \times N)$ is the vector for the degrees of nodes. 
1. for $i:=1$ to $N$ do

2. I $[i]:=i ;$ Degree $[i]:=0 ; \quad \backslash$ Initial vectors

3. endfor;

4. $1:=\operatorname{random}(1, N)$;

II Choose the root

5. R:=I[N];I[N]:=I[I];I[I]:=R; \\Replacement with the last element

6. $\mathrm{m}:=1$

$1 \backslash$ current numbers of nodes in a tree and

5. $\mathrm{k}:=0$;

8. while $\mathrm{m}<\mathrm{N}$ do

$\backslash$ nodes restricted for further choice

9. $\mathrm{s}:=\operatorname{random}(\mathrm{N}-\mathrm{m}+1, \mathrm{~N}-\mathrm{k})$; $\backslash \backslash$ For admissible node in a tree

10. $\mathrm{t}:=\operatorname{random}(1, \mathrm{~N}-\mathrm{m})$; $\backslash \backslash$ For new node

11. IO $[\mathrm{m}]:=\mathrm{I}[\mathrm{t}] ; \mathrm{OU}[\mathrm{m}]:=\mathrm{I}[\mathrm{s}] ; \backslash \backslash$ Registration

12. INC(Degree $[\mathrm{I}[\mathrm{t}]]$ ); INC(Degree $[\mathrm{I}[\mathrm{s}]]$ );

$\backslash \backslash$ Now let's Check for the maximum possible degree

\IIf YES then restrict the node for future choice

13. if Degree $[\mathrm{I}[\mathrm{s}]]=\mathrm{Deg}$ then

14. I $[\mathrm{s}]:=\mathrm{I}[\mathrm{N}-\mathrm{k}] ; \operatorname{INC}(\mathrm{k})$;

15. endif;

16. $\mathrm{R}:=\mathrm{I}[\mathrm{t}]$; $\backslash \backslash$ Rearrange the working vector: from $\mathrm{N}-\mathrm{k}+1$ to $\mathrm{I}[\mathrm{t}]:=\mathrm{I}[\mathrm{N}-\mathrm{m}] ; \backslash \backslash \mathrm{N}$ - nodes restricted for further selection $\mathrm{I}[\mathrm{N}-\mathrm{m}]:=\mathrm{R}$;

17. $\operatorname{INC}(\mathrm{m})$;

\\} \text { Working step is done }

18. endwhile.

Remark: There cannot be a deadlock in this algorithm: as $2 \leq \operatorname{Deg} \leq N-1$, the subset $X_{i}^{*}=\left\{v_{j} \mid v_{j} \in X_{i} \& \operatorname{deg}\left(v_{j}\right)<D e g\right\}$ is always non-empty (at least the last node added to the generated tree always has degree one). And $A_{i+1}$ includes all the edges that connects these nodes with free nodes.

From this we can simply construct the algorithm for generating unbalanced binary tree (B-tree) with the number of nodes $N>4$. Note that node degree in B-tree is limited to 3 . So we can use the following generating scheme:

\section{Algorithm for Generating B-trees.}

Input: $N$ is the number of nodes.

Output: $O U[1 . . N-1]$ and $I O[1 . . N-1]$ are the vectors of numbers starting and ending nodes of the resulting tree edges ( $i$-th edge connect node $O U_{i}$ with $I O_{i}$ ), Root is the number of root node.

1. $\mathrm{N} 1:=\operatorname{random}(1, \mathrm{~N}+1) ; \mathrm{N} 2:=\mathrm{N}-\mathrm{N} 1+1 ;$

2. Call GTD (N1,3,OU1, I01, Degree1);

3. Call GTD (N2,3, OU2, IO2, Degree2);

4. Using vectors Degree1 and Degree2 the nodes $s$ and $t$ are chosen from nodes with degree 1 in both trees, respectively;

5. Renumbering of nodes: the node $s$ becomes node number $\mathrm{N} 1$ in first tree, the node $t$ becomes node number $N 1$ in second tree.

6. Root:=N1;

$\backslash$ Root is assigned

7. for $i:=1$ to $N 1-1$ do 
8. IO $[i]:=\operatorname{IO1}[\mathrm{i}] ; \mathrm{OU}[\mathrm{i}]:=\mathrm{OU} 1[\mathrm{i}] ;$ \} \text { First tree is the left part }

9. endfor;

10. for $i:=N 1$ to $N 1+N 2-1$ do $\quad \backslash$ Second tree is the right part 11. IO [i] :=IO2 [i] +N1-1; OU [i] :=OU2 [i] +N1-1;

12. endfor.

Remark: If we assume the possibility of one-side B-tree then $N 2$ can be 0 . In this case we simply generate one tree with restriction $D e g=3$ and then choose a root from all nodes with a degree of 1 or 2 .

\subsection{Generating Trees with Given Node Degrees}

Now let us have the strict values for $\operatorname{deg}\left(v_{i}\right)$ in the resulting tree. This is more complicated case, because there can be a deadlock in direct use of the basic algorithm. In fact, it is enough that $\sum_{j} \operatorname{deg}\left(v_{j} \mid v_{j} \in X_{i}\right)=2(i-1)$. For example, if some $v_{s}$ and $v_{t}$ both have degree one where $v_{s}$ is selected as a root on the preliminary step, and $e_{s t}$ is the first choice, then we have the deadlock on the very first step of the algorithm. To avoid the deadlock we employ the following rule: each time when we have $\left(\sum_{j} \operatorname{deg}\left(v_{j} \mid v_{j} \in X_{i}\right)=2(i-1)-1\right) \&(i<N-2)$ we add all edges that connects nodes from $X_{i}$ with free nodes with prescribed degree one to the $E x_{i}$.

Let us prove the correctness of this rule based on the proof by contradiction. Assume that $\sum_{j} \operatorname{deg}\left(v_{j} \mid v_{j} \in X_{i}\right)=2(i-1)-1$. The deadlock will take place if and only if next node has degree one. Assume that all free nodes (their number is $N-i$ ) have the prescribed degree one. So the total sum of degrees by all nodes is $2(i-1)-1+N-i=N+i-3$ while for a tree it must be $2(N-1)$. We have a contradiction, so our rule is proved to be correct.

\section{Generating Trees with Different Probabilities of Edge Existence}

This case differs from the previous one only in the procedure of a new edge choice. In the case of equal probability the set $A_{i}$ is virtual and choice is made between nodes, and now we need to have it in some form that allows us to make choice of new edge as a numbered pair of nodes. Note that in general case the sum of probabilities of the edge existence is not equal to 1 as these events are not alternative. Thus for the choice of one edge from the set we use normalized probabilities $p_{i}^{*}=p_{i} / \sum_{j=1}^{n} p_{j}$.

Let us denote $\frac{N(N-1)}{2}$ as $n$. In hereafter we use the presentation of undirected graph with $N$ nodes by the up-diagonal part of its adjacency matrix $V V$ unfolded into a vector of the length $n$. We denote this vector as $D(G)$ or simply $D$. 
Obviously there is one-to-one correspondence between any element $V V_{i j}$ and some element $D_{l}$. Let us derive a formula for $l$ through $i, j$ and $N$.

Each element $V V_{i j}$ has $i-1$ rows above it and $k$-th row contains $N-k$ elements that belong to the up-diagonal part. The $i$-th row contains $j-i-1$ elements to the left of $V V_{i j}$ which belong to this up-diagonal part. Thus

$$
l=[(N-1)+(N-2)+\ldots+(N-i+1)]+j-i=\frac{(2 N-i)(i-1)}{2}+j-i .
$$

To obtain $i$ and $j$ from the values of $l$ and $N$ the following primitive algorithm is provided.

1. procedure RowCol (N,l : integer; var Row,Col: integer);

2. $\operatorname{var} \mathrm{m}$ : integer;

3. begin $i:=1$;

4. while $(i<N)$ and $(m>0)$ do

5. $\mathrm{m}:=\mathrm{m}-\mathrm{N}+\mathrm{i} ; \operatorname{INC}(\mathrm{i})$;

6. endwhile;

7. Row:=i-1;

8. $\mathrm{Col}:=\mathrm{N}+\mathrm{m}$;

9. end RowCol;

Note that if we don't need to save the computer memory it is simpler to use the array of pairs $(i, j)$ instead of transformation described above. Now we go on to the algorithms.

As any node inevitably belongs to the spanning tree, we can choose any one as a root on the preliminary step with equal probability. Then we construct the initial list (vector) of possible edges as list of edges incident to the root.

It's simple to obtain the maximum possible length $L_{m}$ of the list of possible edges: recollect that with each new $i$-th edge chosen we eliminate from it $i-1$ edges that connected new node with the nodes from $X_{i}$ and include $N-i$ edges that connect new node with the nodes from $\bar{X}_{i}$. Thus, after completion the $i$-th step we have

$$
L=(N-1)-1+(N-2)-2+(N-3)-3+\ldots-(i-1)+(N-i)=(N-i) i,
$$

and $\left\lceil L_{m}=N^{2} / 4\right\rceil$. Any additional limitation will only decrease this number.

The pseudo code of the algorithm looks as follows.

\section{Algorithm for Generating Tree with Different Probabilities of Edge} Existence and Limited Maximum Node Degree.

Input: $N>1$ is the number of nodes, $\operatorname{Prob}[1 . . n]$ is the vector of probabilities of edges existence (this vector corresponds to the $\| \varepsilon_{i j}=P\left(e_{i j}\right.$ exists) $\left.\|\right), D e g$ is the possible maximum node degree.

Output: $O U[1 . . N-1]$ and $I O[1 . . N-1]$ are the vectors of the resulting tree edges starting and ending nodes numbers ( $i$-th edge connect node $O U_{i}$ with $I O_{i}$ ), Degrees $(1 \times N)$ is the vector of the nodes degrees.

Working arrays and variables: $X[1 . . N-1]$ is the vector for numbers of nodes that are in a generated tree already, $W[1 . . K], P[1 . . K]$ - vectors of numbers of edges 
admissible for choice on a step and their normalized probabilities, respectively. $M$ is current number of nodes that are in a tree already and $K$ is a number of edges that are admissible for choice on a current step.

1. $\mathrm{n}:=\mathrm{N} *(\mathrm{~N}-1) / 2$; $\quad \backslash$ Whole number of possible edges $1 \backslash$ Initialization of the vector for edge numbers

2. for $i:=1$ to $n$ do $I[i]:=i$; endfor;

$\backslash \backslash$ Initialization of the vector for node degrees

3. for $i:=1$ to $N$ do Degree $[i]:=0$; endfor;

4. $1:=\operatorname{random}(\mathrm{P}, 1, \mathrm{n}) ; \mathrm{X}[1]:=1 ; \backslash \backslash$ Root is selected

5. $S:=0.0 ; j:=0 ; M:=1 ; \backslash \backslash M$ - current number of selected nodes, $\mathrm{K}:=\mathrm{N}-1 ; \quad \backslash \backslash \mathrm{K}$ - current number of free nodes

$\backslash$ Now let's obtain the vector for admissible edges

$\backslash$ and Sum of probabilities for future normalization

6. for $\mathrm{v}:=1$ to $1-1,1+1$ to $\mathrm{N}$ do

7. $\operatorname{INC}(j) ; W[j]:=I[\operatorname{InUpDiag}(j, 1)]$;

8. $\mathrm{S}:=\mathrm{S}+\operatorname{Prob}[\operatorname{InUpDiag}(\mathrm{N}, \mathrm{V}, \mathrm{I})]$;

9. endfor;

10. for $i:=1$ to $N-1$ do

$\backslash$ Probabilities normalization

11. for $v:=1$ to $K$ do $P[v]:=P[v] / S$; endfor;

12. $\mathrm{j}:=\mathrm{W}[\operatorname{random}(P, 1, \mathrm{~m})] ; \operatorname{INC}(M) ; 0 l d S:=S ; \backslash \backslash$ New edge is selected

$\backslash$ Let's obtain the numbers of adjusted nodes and increment

$\backslash$ thier degrees

13. Call RowCol (N,j,s,t); INC(Degree [s]); INC(Degree [t]);

$\backslash$ Now let us define which node is new one

14. $\mathrm{w}:=\mathrm{FindPlace}(\mathrm{s}, \mathrm{X}, \mathrm{OK})$;

15. if $\mathrm{OK}$ then NewNode:=t else NewNode:=s; $\mathrm{s}:=\mathrm{t}$; endif;

$1 \backslash \mathrm{s}$ is the "old" node in the selected edge

16. $\mathrm{X}[\mathrm{M}]:=$ NewNode;

17. if Degree[s]=Deg then $\backslash \backslash$ Check for maximum degree

18. for $t:=1$ to $N$ do

$\backslash$ All free edges connected with a node with maximum possible

$\backslash$ degree must be removed from the set of admissible

19. $\mathrm{w}:=\mathrm{FindPlace}(\mathrm{t}, \mathrm{X}, \mathrm{OK})$;

20. if not $\mathrm{OK}$ then

$$
\mathrm{q}:=\operatorname{InUpDiag}(\mathrm{N}, \mathrm{t}, \mathrm{s}) ; \mathrm{S}:=\mathrm{S}-\mathrm{P}[\mathrm{q}] ; \operatorname{remove}(\mathrm{q}, \mathrm{W}) ; \operatorname{DEC}(\mathrm{K}) \text {; }
$$

21. endif; endfor; endif;

22. \\ Now let us exclude all edge that connects new node with

$\backslash \backslash$ old ones from the set of admissible and include edges that

\I connect new node with free ones into it

23. $\mathrm{SS}:=0.0$;

24. for $\mathrm{v}:=1$ to NewNode-1, NewNode+1 to $\mathrm{N}$ do

25. $\mathrm{t}:=\operatorname{InUpDiag}(\mathrm{X}[\mathrm{v}]$, NewNode); $\mathrm{w}:=\mathrm{FindPlace}(\mathrm{t}, \mathrm{W}, \mathrm{OK})$;

26. if $\mathrm{OK}$ then $\mathrm{S}:=\mathrm{S}-\mathrm{P}[\mathrm{W}] ; \operatorname{remove}(\mathrm{t}, \mathrm{W}) ; \mathrm{DEC}(\mathrm{K})$;

27. else $\mathrm{SS}:=\mathrm{SS}+\operatorname{Prob}[\mathrm{t}] ; \operatorname{INC}(\mathrm{K}) ; \mathrm{W}[\mathrm{K}]:=\mathrm{t}$; 
28. endif endfor;

$\backslash$ Let us recalculate a sum for normalization

29. $\mathrm{S}:=\mathrm{SS}+\mathrm{S} * 01 \mathrm{dS}$;

30. endfor.

The procedure InUpDiag (line 8) is for expression (5).

\section{Conclusion}

We have presented general methods and computer algorithms for generating random trees with many essential properties. It is shown that the use of trial method is mostly ineffective. Meanwhile the time complexity of the proposed algorithms based on the consequential tree growth are proven to be effective in terms of the number of operations and the memory use. Random trees are widely used in different applications by themselves, and furthermore can be efficiently employed for generating various connected graphs. As graphs used in applications have different limitations, their spanning trees must satisfy them too. Our basic algorithms allow those. In the future we present algorithms for generating connected graphs and graphs "similar to real nets." The last task assumes definitions of some special limitations that are typical for such graphs.

\section{References}

1. M. Doar, Multicast in the ATM environment. PhD thesis, Cambridge Univ., Computer Lab., September 1993.

2. M. Doar, "A Better Mode for Generating Test Networks," Proc. Global Telecommunication Conf. GLOBECOM'96, pp. 86-93, 1996.

3. Chai-Keong Toh, "Performance Evaluation of Crossover Switch Discovery Algorithms for Wireless ATM LANs," Proc. of INFOCOM'96, pp. 1380-1387, 1993.

4. A.S. Rodionov, L.N. Postnikova, and O.K. Rodionova, "Program Complex for Graph Generating in the GRAPH-ES Package," Materials of the Conf. "Computers and System Analysis," Novosibirsk, 1982, pp. 13-15., 1982. (in Russian)

5. A.S. Rodionov, "Graph Generating in the GRAPH-ES Package," Materials of the Conf. "Methods and Programs for Solving Optimization Problems on Graphs and Nets," Novosibirsk, 1982, Part 1, pp. 170-171., 1982. (in Russian)

6. M.I. Netchepurenko, V.K. Popkov, S.M. Mainagashev, etc, Algorithms and Programs for Solving Optimization Problems on Graphs and Networks. Novosibirsk, Nauka P.A., 1990, 515 p. (in Russian).

7. G. Omarova, "Program Complex for Graph Generating," Proc. of Young Scientists Annual Conf., Institute of Computational Mathematics and Mathematical Geophysics of Siberian Division of the Russian Academy of Science, Novosibirsk, pp. 174-181, 1998. (in Russian) 